\title{
Centers and limit cycles of a generalized cubic Riccati system
}

\author{
Zhengxin Zhou ${ }^{\mathrm{a}}$, Valery G. Romanovskib ${ }^{\mathrm{b}, \mathrm{d}}$, Jiang $\mathrm{Yu}^{\mathrm{e}, *}$ \\ ${ }^{a}$ School of Mathematical Sciences, Yangzhou University, Yangzhou 225002, P. R. China \\ ${ }^{b}$ Faculty of Electrical Engineering and Computer Science, University of Maribor \\ Smetanova 17, Maribor, SI-2000 Maribor, Slovenia \\ ${ }^{c}$ CAMTP - Center for Applied Mathematics and Theoretical Physics, \\ University of Maribor, Krekova 2, SI-2000 Maribor, Slovenia \\ ${ }^{d}$ Faculty of Natural Science and Mathematics, University of Maribor, Koroška c. 160, SI-2000 Maribor, Slovenia \\ ${ }^{e}$ School of Mathematical Sciences, Shanghai Jiao Tong University, Shanghai 200240, P. R. China
}

\begin{abstract}
We obtain condition for existence of a center for a cubic planar differential system, which can be considered as a polynomial subfamily of the generalized Riccati system. We also investigate bifurcations of small limit cycles from the components of the center variety of the system.
\end{abstract}

Keywords: center, limit cycle, cyclicity

2010 MSC: $34 \mathrm{C} 05,34 \mathrm{C} 07$

\section{Introduction}

Consider systems of ordinary differential equations on $\mathbb{R}^{2}$ of the form

$$
\dot{x}=P(x, y), \quad \dot{y}=Q(x, y),
$$

where $P$ and $Q$ are polynomials, $\max \{\operatorname{deg} P, \operatorname{deg} Q\}=n$. We view (1) as defining a family of systems parametrized by the coefficients of $P$ and $Q$. That is, the degree of polynomials $P$ and $Q$ in system (1) is fixed and the coefficients of the polynomials are parameters $\lambda_{1}, \ldots, \lambda_{N}$, so the $N$-tuple of the parameters $p=\left(\lambda_{1}, \ldots, \lambda_{N}\right)$ is a point in a Euclidean $N$-dimensional space $\mathcal{E}$, and we identify each point in $\mathcal{E}$ with its corresponding system (1). In this paper the space $\mathcal{E}$ of parameters is either $\mathbb{R}^{N}$ or $\mathbb{C}^{N}$.

A singular point $\left(x_{0}, y_{0}\right)$ of real system (1) is a stable focus if every nearby trajectory spirals towards to it, an unstable focus if every nearby trajectory spirals away from it, and it is a center if every nearby trajectory is an oval.

A singular point $\left(x_{0}, y_{0}\right) \in \mathbb{R}^{2}$ of a system $p \in \mathcal{E} \subset \mathbb{R}^{N}$ is said to have cyclicity $k$ with respect to $\mathcal{E}$ if and only if any sufficiently small perturbation of $p$ in $\mathcal{E}$ has at most $k$ limit cycles in a sufficiently small neighborhood of $\left(x_{0}, y_{0}\right)$, and $k$ is the smallest number with this property. The problem of cyclicity of a center or a focus of a system of the form (1) is known as the local 16th Hilbert problem in (Françoise and Yomdin (1997)), based on its connection to Hilbert's

${ }^{*}$ Corresponding author

Email addresses: zxzhou@yzu.edu.cn (Zhengxin Zhou), valery.romanovsky@uni-mb.si (Valery

G. Romanovski), jiangyu@sjtu.edu.cn (Jiang Yu)

Preprint submitted to Elsevier

July 14, 2018 
still unresolved 16th problem, which in part asks for a bound on the number of limit cycles of system (1) in terms of degree $n$ of the system (see, e.g. survey papers Giné (2007); Li (2003)).

The problem of cyclicity is closely connected to the center problem, that is, the problem of finding systems with centers in a given polynomial family (1). The studies of the center problem dates back to 1908 when Dulac (1908) investigated the case of the quadratic system. The literature devoted to the subject is vast, see e.g. Amel'kin et al. (1982); Christopher and Li (2007); Liu et al. (2008); Romanovski and Shafer (2009); Sibirskii (1976) and the references that they contain.

After a linear transformation and time rescaling any planar polynomial system (1) with an elementary center or weak focus can be written in the form

$$
\dot{x}=-y+\widetilde{P}(x, y), \quad \dot{y}=x+\widetilde{Q}(x, y),
$$

where $\widetilde{P}$ and $\widetilde{Q}$ are polynomials without free and linear terms. By the Poincare-Lyapunov theorem for real system (2) the origin is a center if and only if in a neighborhood of the origin the system admits a real analytic local first integral of the form

$$
\Psi(x, y)=x^{2}+y^{2}+\sum_{j+k=3} \psi_{j k} x^{j} y^{k} .
$$

Recently Llibre and Valls (2014, 2015) investigated the planar system

$$
\dot{x}=f(y), \quad \dot{y}=g_{2}(x) y^{2}+g_{1}(x) y+g_{0}(x),
$$

which is called the generalized Riccati system (4), since it becames the classical Riccati system if $f(x) \equiv 1$.

In this paper we consider a particular subfamily of the generalized Riccati system, namely, the cubic system

$$
\begin{aligned}
\dot{x} & =-y+a_{02} y^{2}, \\
\dot{y} & =\left(b_{02}+b_{12} x\right) y^{2}+\left(b_{11} x+b_{21} x^{2}\right) y+\left(b_{20} x^{2}+b_{30} x^{3}+x\right) \\
& =x+b_{20} x^{2}+b_{11} x y+b_{02} y^{2}+b_{30} x^{3}+b_{21} x^{2} y+b_{12} x y^{2},
\end{aligned}
$$

where $a_{i j}, b_{k s}$ are real or complex parameters. We first find conditions for system (5) with complex parameters to have an analytic first integral of the form (3), so, the corresponding real systems have a center at the origin. Then, we study limit cycles bifurcations from the centers of real systems (5).

\section{Preliminaries}

For system (2) it is always possible to find a function

$$
\Phi(x, y)=x^{2}+y^{2}+\sum_{j+k=3}^{\infty} \phi_{j k} x^{j} y^{k},
$$

such that

$$
\frac{\partial \Phi}{\partial x}(-y+\widetilde{P}(x, y))+\frac{\partial \Phi}{\partial y}(x+\widetilde{Q}(x, y))=\sum_{m=2}^{\infty} g_{m-1}\left(u^{2}+v^{2}\right)^{m} .
$$


The coefficients $g_{k}$ in (7) are polynomials in parameters of system (7) called the focus quantities of the system. Each polynomial $g_{i}$ represents an obstacle for existing of integral (3), that is, system (2) admits an integral (3) if and only if $g_{1}=g_{2}=g_{3}=\cdots=0$. Thus, the set of all systems in the parametric family (2) with centers (equivalently, the set of systems with a first integral of the form (3) is the variety $1 V^{\mathbb{R}} \subset \mathbb{R}^{n}$ of the ideal $B=\left\langle g_{1}, g_{2}, g_{3}, \ldots\right\rangle$. By the Hilbert Basis Theorem there is an integer $k$ that $B=\left\langle g_{1}, \ldots, g_{k}\right\rangle$, however there are no regular methods to find such $k$.

We will also consider system (2) as a system with complex parameters. In such case the polynomials $g_{i}$ in (7) are polynomials with complex coefficients and then their variety is a complex variety, which we will denote $V^{\mathbb{C}}$. All systems whose parameters belong to the variety $V^{\mathbb{C}}$ admit local analytic first integral of the form (3). We call $V^{\mathbb{R}}$ and $V^{\mathbb{C}}$ the real and complex center varieties of system (2).

It was shown in García et al. (2016) that knowing the complex variety of a real polynomial system can be helpful for investigation of cyclicity of the real system. We also need to work with complex varieties to apply our computational approach for computing conditions of integrability.

To find a real or complex center variety of a polynomial system (2), one computes a few first focus quantities $g_{1}, \ldots, g_{k}$ of the system and then finds decomposition of the variety of the ideal $B_{k}=\left\langle g_{1}, \ldots, g_{k}\right\rangle$ obtaining the necessary conditions of existence of integral (3). Then it is necessary to prove the sufficiency of the obtained condition. We recall that one of most powerful methods to prove the existence of integral (3) is the Darboux method, which allows to construct a first integral or integrating factor using Darboux polynomials (see, e.g. surveys Llibre (2011); Llibre and Zhang (2012)). A Darboux polynomial of system (1) is a polynomial $f(x, y)$ satisfying

$$
\frac{\partial f}{\partial x} P+\frac{\partial f}{\partial y} Q=K f
$$

where $K(x, y)$ is a polynomial called the cofactor of $f$. It is easy to see that if $f$ is a Darboux polynomial of system (1), then $f=0$ is an algebraic invariant curve of the system.

It is easy to see that if system (2) has $p$ irreducible Darboux polynomials $f_{1}, \ldots, f_{p}$ with the associated cofactors $K_{1}, \ldots, K_{p}$, such that $s_{1} K_{1}+\ldots+s_{p} K_{p}=0$, then the function $H=f_{1}^{s_{1}} \ldots f_{p}^{s_{p}}$ is a first integral of the system (called the Darboux first integral) and if

$$
s_{1} K_{1}+\ldots+s_{p} K_{p}=\frac{\partial P}{\partial x}+\frac{\partial Q}{\partial y},
$$

then the function $\mu=f_{1}^{s_{1}} \ldots f_{p}^{s_{p}}$ is an integrating factor of (1) (called the Darboux integrating factor).

\section{Itegrability conditions for system (5)}

In this section we assume that parameters of system (5) are complex and find the complex variety $V^{\mathbb{C}}$ of the system. The study yields that the variety consists of 7 irreducible components.

Theorem 1. System (5) has an analytic integral of the form (3) if the 7-tuple $\left(a_{02}, b_{20}, b_{11}, b_{12}, b_{02}\right.$, $\left.b_{21}, b_{30}\right)$ of its parameters belong to the variety of one of the following prime ideals:

\footnotetext{
${ }^{1}$ We remind that the variety of a given ideal $F$ generated by polynomials $f_{1}\left(x_{1}, \ldots, x_{m}\right), \ldots, f_{s}\left(x_{1}, \ldots, x_{m}\right)$ over a field $k, F=\left\langle f_{1}, \ldots, f_{s}\right\rangle \subset k\left[x_{1}, \ldots, x_{m}\right]$, is the set $\mathbf{V}(F)=\left\{\left(x_{1}, \ldots, x_{n}\right) \in k^{n} \mid f\left(x_{1}, \ldots, x_{m}\right)=0\right.$ for all $\left.f \in F\right\}$. 
$I_{1}=\left\langle b_{21}, b_{20}, b_{02}\right\rangle$,

$I_{2}=\left\langle b_{30}, b_{12}, b_{02}, b_{11} b_{20}-b_{21}\right\rangle$,

$I_{3}=\left\langle b_{30}, b_{21}, b_{12},-2 b_{02} b_{11}^{2}+4 b_{02}^{2} b_{20}-b_{11}^{2} b_{20}, 2 a_{02} b_{11}+b_{11}^{2}-4 b_{02} b_{20}, 2 a_{02} b_{02}-b_{02} b_{11}-\right.$ $\left.b_{11} b_{20}, 4 a_{02}^{2}-b_{11}^{2}-4 b_{20}^{2}\right\rangle$,

$I_{4}=\left\langle b_{21}, b_{11}, a_{02}\right\rangle$,

$I_{5}=\left\langle a_{02}, b_{02} b_{21}+b_{11} b_{30}, 2 b_{02} b_{12}+b_{12} b_{20}+b_{02} b_{30}, b_{02} b_{11}+b_{11} b_{20}-b_{21}, b_{02}^{2}+b_{02} b_{20}+b_{30}, b_{12} b_{20} b_{21}-\right.$

$2 b_{11} b_{12} b_{30}-b_{11} b_{30}^{2}, b_{11} b_{20} b_{21}-b_{21}^{2}-b_{11}^{2} b_{30}, b_{12} b_{20}^{2}-4 b_{12} b_{30}-b_{02} b_{20} b_{30}-2 b_{30}^{2}, b_{11} b_{12} b_{20}-$

$\left.2 b_{12} b_{21}+b_{11} b_{20} b_{30}-b_{21} b_{30},-\left(b_{12} b_{21}^{2}\right)+b_{11}^{2} b_{12} b_{30}+b_{11}^{2} b_{30}^{2}\right\rangle$

$I_{6}=\left\langle b_{21}, b_{12}, b_{11}, b_{02}\right\rangle$,

$I_{7}=\left\langle b_{21}, b_{12}, b_{30}, 3 b_{02}+5 b_{20}, 5 a_{02}-b_{11}, 6 b_{11}^{2}+25 b_{20}^{2}\right\rangle$.

That is, the variety $V^{\mathbb{C}}$ of system has the irreducible decomposition $V^{\mathbb{C}}=\cup_{k=1}^{7} V_{k}^{c}$, where $V_{k}^{c}=\mathbf{V}\left(I_{k}\right)(k=1, \ldots, 7)$.

Proof. Necessity. For system (5) we have computed eight first focus quantities $g_{1}, g_{2}, \ldots, g_{8}$ and then tried to find the irreducible decomposition of the variety $\mathbf{V}(I)$ of the ideal

$$
I=\left\langle g_{1}, g_{2}, \ldots, g_{8}\right\rangle
$$

over the field of rational numbers using the routine minAssGTZ Decker et al. (2010) (which is based on the algorithm of Gianni et al. (1988)) of the computer algebra system Singular Decker et al. (2012), but due to high complexity of calculations we have not succeeded to complete them with our computational facilities. However computing in the polynomial ring

$$
\mathbb{Z}_{32003}\left[a_{02}, b_{02}, b_{11}, b_{12}, b_{20}, b_{21}, b_{30}\right. \text { 目 }
$$

using the degree reverse lexicographic ordering with $a_{02}>b_{02}>b_{11}>b_{12}>b_{20}>b_{30}>b_{21}$ we have found that in the affine space $\mathbb{Z}_{32003}^{7}$ the variety of $I$ consists of 8 irreducible components defined by the following 8 prime ideals:

$\tilde{I}_{1}=\left\langle b_{21}, b_{20}, b_{02}\right\rangle$,

$\tilde{I}_{2}=\left\langle b_{30}, b_{12}, b_{02}, b_{11} b_{20}-b_{21}\right\rangle$

$\tilde{I}_{3}=\left\langle b_{30}, b_{21}, b_{12}, a_{02} b_{11}-16001 b_{11}^{2}-2 b_{02} b_{20}, a_{02} b_{02}+16001 b_{02} b_{11}+16001 b_{11} b_{20}, a_{02}^{2}-8001 b_{11}^{2}-\right.$ $\left.b_{20}^{2}, b_{02} b_{11}^{2}-2 b_{02}^{2} b_{20}-16001 b_{11}^{2} b_{20}\right\rangle$,

$\tilde{I}_{4}=\left\langle b_{21}, b_{11}, a_{02}\right\rangle$,

$\tilde{I}_{5}=\left\langle a_{02}, b_{02} b_{21}+b_{11} b_{30}, b_{02} b_{12}-16001 b_{12} b_{20}-16001 b_{02} b_{30}, b_{02} b_{11}+b_{11} b_{20}-b_{21}, b_{02}^{2}+b_{02} b_{20}+\right.$ $b_{30}, b_{12} b_{20} b_{21}-2 b_{11} b_{12} b_{30}-b_{11} b_{30}^{2}, b_{11} b_{20} b_{21}-b_{11}^{2} b_{30}-b_{21}^{2}, b_{12} b_{20}^{2}-b_{02} b_{20} b_{30}-4 b_{12} b_{30}-$ $\left.2 b_{30}^{2}, b_{11} b_{12} b_{20}+b_{11} b_{20} b_{30}-2 b_{12} b_{21}-b_{21} b_{30}, b_{11}^{2} b_{12} b_{30}+b_{11}^{2} b_{30}^{2}-b_{12} b_{21}^{2}\right\rangle$,

$\tilde{I}_{6}=\left\langle b_{21}, b_{12}, b_{11}, b_{02}\right\rangle$,

$\tilde{I}_{7}=\left\langle b_{30}, b_{21}, b_{12}, b_{11}-15273 b_{20}, b_{02}-10666 b_{20}, a_{02}+3346 b_{20}\right\rangle$,

$\tilde{I}_{8}=\left\langle b_{30}, b_{21}, b_{12}, b_{11}+15273 b_{20}, b_{02}-10666 b_{20}, a_{02}-3346 b_{20}\right\rangle$.

Lifting these ideals into the ring $\mathbb{Q}\left[a_{02}, b_{02}, b_{11}, b_{12}, b_{20}, b_{21}, b_{30}\right]$ using the rational reconstruction algorithm of Wang et al. (1982) we obtain the ideals $I_{1}, \ldots, I_{6}$ given in the statement of Theorem 1 and the ideals

$\hat{I}_{7}=\left\langle b_{30}, b_{21}, b_{12}, b_{11}+\frac{51}{44} b_{20}, b_{02}+\frac{5}{3} b_{20}, a_{02}+\frac{161}{67} b_{20}\right\rangle$

and

$\hat{I}_{8}=\left\langle b_{30}, b_{21}, b_{12}, b_{11}-\frac{51}{44} b_{20}, b_{02}+\frac{5}{3} b_{20}, a_{02}-\frac{161}{67} b_{20}\right\rangle$.

${ }^{2}$ It appear, for the first time modular computations were used for studies on the center and cyclicity problems in Edneral (1997) 
To check the correctness of the obtained decomposition we use the procedure proposed in Romanovski and Prešern (2011).

First, using the Radical Membership Tes ${ }^{3}$ we check if all focus quantities $g_{i}(i=1, \ldots, 8)$ vanish on each of the varieties $\mathbf{V}\left(I_{1}\right), \ldots, \mathbf{V}\left(I_{6}\right), \mathbf{V}\left(\hat{I}_{7}\right), \mathbf{V}\left(\hat{I}_{8}\right)$. The calculations show that all polynomials $g_{i}$ are equal to zero on each of varieties $\mathbf{V}\left(I_{1}\right), \ldots, \mathbf{V}\left(I_{6}\right)$, but not on the varieties $\mathbf{V}\left(\hat{I}_{7}\right), \mathbf{V}\left(\hat{I}_{8}\right)$. This means, that $\mathbf{V}\left(\hat{I}_{7}\right)$ and $\mathbf{V}\left(\hat{I}_{8}\right)$ are not correct components of the irreducible decomposition of $\mathbf{V}(I)$. A usual recipe to find the correct components of the decomposition in such situation is to recompute the decomposition over a few fields of larger characteristics (see e.g. Arnold (2003)). However instead of doing this we observe that both ideals $\tilde{I}_{7}$ and $\tilde{I}_{8}$ contain the polynomials $b_{30}, b_{21}, b_{12}$ and compute with minAssGTZ of SINGULAR the minimal associate primes of the ideal $\left\langle I, b_{30}, b_{21}, b_{12}\right\rangle$ over the field $\mathbb{Q}$ obtaining the component defined by the ideal $I_{7}$ of the statement of the theorem.

Now, to check the correctness of the obtained conditions we computed the ideal $\tilde{I}=\cap_{s=1}^{7} I_{s}$, which defines the union of all seven components listed in the statement of the theorem and have checked that reduced Gröbner bases of all ideals $\left\langle\tilde{I}, 1-w g_{k}\right\rangle$ (where $k=1, \ldots, 8$ and $w$ is a new variable) computed over $\mathbb{Q}$ are $\{1\}$. By the Radical Membership Test it means that

$$
\mathbf{V}(\tilde{I}) \subset \mathbf{V}(I) .
$$

To check the opposite inclusion it is sufficient to check that

$$
\langle I, 1-w f\rangle=\langle 1\rangle
$$

for all polynomials $f$ from a basis of $\tilde{I}$. Unfortunately, we were not able to perform the check over the field $\mathbb{Q}$ however we have checked that (10) holds over a few fields of finite characteristic. It yields that (10) holds with high probability Arnold (2003).

Sufficiency. We now prove that if the coefficients of the system belong to one of varieties mentioned in the statement of the theorem then the system has an analytic first integral of the form (3).

Usually the center variety of a polynomial system contains components corresponding to Hamiltonian, time-reversible and Darboux integrable systems.

It is easy to see that systems from $V_{6}^{\mathbb{C}}$ are Hamiltonian with the Hamiltonian

$$
H=\frac{x^{2}+y^{2}}{2}+\frac{b_{20} x^{3}}{3}+\frac{b_{30} x^{4}}{4}-\frac{a_{02} y^{3}}{3} .
$$

All time-reversible cubic systems were found in Sibirskii (1976); Jarrah et al. (2001). To use the results of Sibirskii (1976); Jarrah et al. (2001) we first complexify system (5) introducing the variable $z=x+i y$ and obtain from (5) after rescaling of time by $i$ the complex differential equation

$$
\begin{gathered}
\dot{z}=z+\frac{1}{4} z^{2}\left(i a_{02}-b_{02}-i b_{11}+b_{20}\right)-\frac{1}{2} z \bar{z}\left(i a_{02}-b_{02}-b_{20}\right)+\frac{1}{4} \bar{z}^{2}\left(i a_{02}-b_{02}+i b_{11}+b_{20}\right) \\
-\frac{1}{8} z^{3}\left(b_{12}+i b_{21}-b_{30}\right)+\frac{1}{8} z^{2} \bar{z}\left(b_{12}-i b_{21}+3 b_{30}\right)+\frac{1}{8} z \bar{z}^{2}\left(b_{12}+i b_{21}+3 b_{30}\right)-\frac{1}{8} \bar{z}^{3}\left(b_{12}-i b_{21}-b_{30}\right) .
\end{gathered}
$$

\footnotetext{
${ }^{3}$ The test says that for a polynomial $f$ and an ideal $I=\left\langle f_{1}, \ldots, f_{m}\right\rangle$ in $\left.\mathbb{C}\left[x_{1}, \ldots, x_{n}\right] f\right|_{\mathbf{V}(I)} \equiv 0$ if and only if the reduced Gröbner basis of the ideal $\left\langle 1-w f, f_{1}, \ldots, f_{m}\right\rangle$ (here $w$ is a new variable) is equal to \{1\}, see e.g. Cox et al. (1997); Romanovski and Shafer (2009) for more details
} 
Substituting the coefficients of this differential equations into polynomials of Theorem 6 of Jarrah et al. (2001), which define the variety of all time-reversible cubic systems, and then computing with minAssGTZ of SINGULAR the minimal associate primes of the obtained ideal we get the components $V_{1}^{c}, V_{3}^{c}$ and $V_{4}^{c}$ of the statement of the theorem. Hence, all systems from the components $V_{1}^{c}, V_{3}^{c}$ and $V_{1}^{c}$ are time-reversible and, therefore, admit a first integral of the form (3) (see e.g. Romanovski and Shafer (2009) for more details).

Thus, there remains to prove integrability of systems from the components $V_{2}^{c}, V_{5}^{c}$ and $V_{7}^{c}$.

Systems from the component $V_{2}^{c}$, have the form

$$
\dot{x}=-y+a_{02} y^{2}, \quad \dot{y}=x+b_{20} x^{2}+b_{11} x y+b_{11} b_{20} x^{2} y .
$$

Computing we obtain that system (11) admits the Darboux polynomial $f=1+b_{11} y$ with the cofactor $K=b_{11} x\left(1+b_{20} x\right)$. Thus, the function $\mu=\frac{1}{f}$ is a Darboux integrating factor of 111, which allows to construct the analytic first integral

$\Psi=\frac{1}{3}\left(\frac{3 b_{11} y\left(2\left(a_{02}+b_{11}\right)-a_{02} b_{11} y\right)-6\left(a_{02}+b_{11}\right) \log \left(b_{11} y+1\right)}{b_{11}^{3}}+x^{2}\left(2 b_{20} x+3\right)\right)=x^{2}+y^{2}+\ldots$

Systems from $V_{5}^{\mathbb{C}}$ are the so-called reduced Kukles systems. The center problem for such systems has been solved in Jin et al. (1990); Christopher and Lloyd (1990), so by the results of these papers systems from $V_{5}^{\mathbb{C}}$ admit first integral of the form (3).

Finally, system from the component $V_{7}^{\mathbb{C}}$ are of the form

$$
\dot{x}=-y \pm \frac{i b_{20} y^{2}}{\sqrt{6}}, \quad \dot{y}=x+b_{20} x^{2} \pm \frac{5 i b_{20} x y}{\sqrt{6}}-\frac{5 b_{20} y^{2}}{3}
$$

The system has the Darboux polynomial

$$
f=1+\frac{b_{20}^{2} x^{2}}{3}-\frac{b_{20}^{2} y^{2}}{18}+\frac{4 b_{20} x}{3} \pm \frac{1}{3} i \sqrt{\frac{2}{3}} b_{20}^{2} x y \pm i \sqrt{\frac{2}{3}} b_{20} y
$$

with the cofactor $K= \pm \frac{1}{3} i b_{20}(\sqrt{6} x+4 i y)$ which allows to construct the integrating factor $\mu=$ $f^{-5 / 2}$.

Remark. In the statement of theorem the ideals are presented as returned by the routine minAssGTZ of Singular. However looking for Gröbner bases of $I_{5}$ with different ordering of variables we find that the ideal $I_{5}$ is the same as the ideal

$$
\hat{I}_{5}=\left\langle b_{02}^{3}+b_{02}^{2} b_{20}-2 b_{02} b_{12}-b_{12} b_{20},-b_{02} b_{11}-b_{11} b_{20}+b_{21}, b_{02}^{2}+b_{02} b_{20}+b_{30}\right\rangle .
$$

It is easy to see that the conditions defined by these polynomials are equivalent to conditions (iv) of Theorem 3.6 of Christopher and Lloyd (1990).

\section{Limit cycle bifurcations in system (5)}

In this section we study bifurcations of limit cycles from each component of the real center variety of system (5) under perturbations inside the family. It is obvious that $V_{7}^{\mathbb{C}}$ is the empty set in $\mathbb{R}^{7}$. So the real variety $V^{\mathbb{R}}$ of (5) consist of 6 components, $V^{\mathbb{R}}=\cup_{k=1}^{6} V_{k}$, where $V_{k}$ is the set $V_{k}^{\mathbb{C}}$ of Theorem 1 restricted to $\mathbb{R}^{7}$. 
Let $I=\left\langle f_{1}, \ldots, f_{m}\right\rangle \subset k\left[x_{1}, \ldots, x_{n}\right]$ be an ideal and $\mathbf{V}(I)$ be its variety, Assume that a decomposition of $V=\mathbf{V}(I)$ is known and let $p$ be a point from $V$. The tangent space to $V$ at $p$ is defined as $T_{p}=p+\left\{v \mid J_{p}(I) v=0\right\}$, where $J(I)$ is the Jacobian of the polynomials $f_{1}, \ldots, f_{m}$ and $J_{p}$ indicates that it is evaluated at $p$. It follows that $\operatorname{dim} T_{p}=n-\operatorname{rank}\left(J_{p}(I)\right)$. It is said that $p$ is a smooth point of $V$ if $\operatorname{dim} T_{p}=\operatorname{dim} V_{p}$. Let $C$ be a component of $V$ of codimension $k$ and assume that $p \in C, \operatorname{rank}\left(J_{p}(I)\right)=s$. Then $k \geq s$ and $p$ is a smooth point $C$ if and only if $k=s$; in this case $\operatorname{rank}\left(J_{q}(I)\right)=k$ at any smooth point of $C$.

The following statement is proved by Christopher (2005).

Theorem 2. Assume that for system (2) $p \in K$ is a point on the center variety and that the first $k$ of the focus quantities $g_{i}$ have independent linear parts. Then $p$ lies on a component of the center variety of codimension at least $k$ and there are bifurcations of (2) which produce $k$ limit cycles locally from the center corresponding to the parameter value $p$.

If, furthermore, we know that $p$ lies on a component of the center variety of codimension $k$, then $p$ is a smooth point of the variety, and the cyclicity of the center for the parameter value $p$ is exactly $k-1$.

In the latter case, $k-1$ is also the cyclicity of a generic point on this component of the center variety.

According to the theorem in some cases the cyclicity of generic point of a component of the center variety can be easily determined if we know the dimension of the components of center variety. The dimension of a complex variety can be computed using algorithms of computational algebra, since it is equal to the degree of the affine Hilbert polynomial of any ideal defining the variety. However determining dimensions of real varieties is more difficult problem. Nevertheless, it is not difficult to determine the dimension of the components of the center variety of real system (2) and to prove the following result.

Theorem 3. The cyclicities of generic point of the components $V_{1}, V_{2}, V_{4}, V_{5}, V_{6}$ of system (5) are 2,3, 2, 3, 3 respectively. The cyclicity of generic point of $V_{3}$ is at least 2.

Proof. It is clear that the codimension of the component $V_{1}$ is 3 . Computing the minors of the matrix $J\left(g_{1}, g_{2}, g_{3}\right)$ evaluated on $V_{1}$ we see that for any point of $p \in V_{1}$ there is a non-zero 3-minor if $f(p) \neq 0$, where

$f=-140 a_{02}^{6} b_{12}+61 a_{02}^{4} b_{11}^{2} b_{12}+9 a_{02}^{3} b_{11}^{3} b_{12}-2 a_{02}^{2} b_{11}^{4} b_{12}-36 a_{02}^{4} b_{12}^{2}+9 a_{02}^{2} b_{11}^{2} b_{12}^{2}+600 a_{02}^{5} b_{11} b_{30}+$ $450 a_{02}^{4} b_{11}^{2} b_{30}+45 a_{02}^{3} b_{11}^{3} b_{30}-15 a_{02}^{2} b_{11}^{4} b_{30}-100 a_{02}^{4} b_{12} b_{30}+64 a_{02}^{3} b_{11} b_{12} b_{30}+37 a_{02}^{2} b_{11}^{2} b_{12} b_{30}+$ $11 a_{02} b_{11}^{3} b_{12} b_{30}+12 a_{02} b_{11} b_{12}^{2} b_{30}-6 b_{11}^{2} b_{12}^{2} b_{30}+750 a_{02}^{3} b_{11} b_{30}^{2}+240 a_{02}^{2} b_{11}^{2} b_{30}^{2}-15 a_{02} b_{11}^{3} b_{30}^{2}-$ $90 a_{02} b_{11} b_{12} b_{30}^{2}+18 b_{11}^{2} b_{12} b_{30}^{2}+210 a_{02} b_{11} b_{30}^{3}$..

Therefore, by Theorem 2 the cyclicity of a generic point of $V_{1}$ is two (more precisely, the cyclicity of point $p \in V_{1}$ is two if $\left.f(p) \neq 0\right)$.

Similar consideration and conclusion are valid for the component $V_{4}$.

It is also obvious that the codimensions of the components $V_{2}$ and $V_{6}$ is 4 and the computations show that the rank of $J\left(g_{1}, \ldots, g_{4}\right)$ at a generic point of the component is 4 , so by Christopher's theorem the cyclicity of generic point of the component is 3 .

To find dimensions of components $V_{3}$ and $V_{5}$ we look for their parametrizations. The component $V_{5}$ can be parametrized as follows:

$$
a_{02}=0, b_{20}=-\frac{t_{2}\left(t_{2}^{2}-2 t_{3}\right)}{t_{2}^{2}-t_{3}}, b_{30}=-\frac{t_{2}^{2} t_{3}}{t_{2}^{2}-t_{3}}, b_{11}=t_{1}, b_{21}=\frac{t_{1} t_{2} t_{3}}{t_{2}^{2}-t_{3}}, b_{02}=t_{2}, b_{12}=t_{3} .
$$


To check this we eliminate from the ideal

$$
\left\langle 1-w\left(t_{2}^{2}-t_{3}\right), a_{02}, b_{20}+\frac{t_{2}\left(t_{2}^{2}-2 t_{3}\right)}{t_{2}^{2}-t_{3}}, b_{30}+\frac{t_{2}^{2} t_{3}}{t_{2}^{2}-t_{3}}, b_{11}-t_{1}, b_{21}-\frac{t_{1} t_{2} t_{3}}{t_{2}^{2}-t_{3}}, b_{02}-t_{2}, b_{12}-t_{3}\right\rangle
$$

of the ring $\mathbb{R}\left[w, t_{1}, t_{2}, t_{3}, a_{02}, b_{20}, b_{30}, b_{11}, b_{21}, b_{12}, b_{02}\right]$ the variables $w, t_{1}, t_{2}, t_{3}$ obtaining the ideal $I_{5}$ from the statement of Theorem 11. By Theorem 2 of (Cox et al., 1997, §3.3) it means that (14) gives a rational parametrization of the variety $\mathbf{V}\left(I_{5}\right)$. Now it is easy to conclude that the dimension of the component is 3 and, hence, the codimension is 4 . Checking that the rank of $J\left(g_{1}, \ldots, g_{4}\right)$ is four almost everywhere on $V_{5}$ we obtain by Theorem 2 that the cyclicity of generic point of the component is 3 .

A parametrization of $V_{3}$ is given by

$$
a_{02}=-\frac{t_{1}\left(t_{1}^{2}+4 t_{2}^{2}\right)}{2\left(t_{1}-2 t_{2}\right)\left(t_{1}+2 t_{2}\right)}, b_{20}=\frac{2 t_{1}^{2} t_{2}}{4 t_{2}^{2}-t_{1}^{2}}, b_{30}=0, b_{11}=t_{1}, b_{21}=0, b_{02}=t_{2}, b_{12}=0 .
$$

From the parametrization we see that the component is two-dimensional, thus, it is of codimension five. However only first three focus quantities have independent linear parts, so we cannot get an upper bound for cyclicity using Theorem 2 and only conclude that the cyclicity is at least two.

Remark. Of course, it is often happens that there many parametrizations of the same variety. Another parametrization of $V_{5}$ can be easily obtained using the polynomial basis of $I_{5}$ presented in (13).

\section{Acknowledgments}

The first author is supported by the NSF of China under grant 11571301 and the NSF of province Jiangsu under grant BK20161327. The second author is supported by the Slovenian Research Agency (ARRS, program P1-0306). The third author is partially supported by the state key program of NNSF of China grant number 11431008, NSF of Shanghai grant number 15ZR1423700.

\section{References}

V.V. Amel'kin, N.A. Lukashevich, A.P. Sadovskii. Nonlinear Oscillations in Second Order Systems. (Russian) Minsk: Belarusian State University, 1982.

E. A. Arnold. Modular algorithms for computing Gröbner bases. J. Symbolic Comput. 35 (2003) 403-419.

C. Christopher. Estimating limit cycles bifurcations. In: Trends in Mathematics, Differential Equations with Symbolic Computations (D. Wang and Z. Zheng, Eds.), 23-36. Basel: Birkhäuser-Verlag, 2005.

C. Christopher and C. Li. Limit Cycles of Differential Equations. Basel: Birkhäuser-Verlag, 2007.

C.J. Christopher, N.G. Lloyd. On the paper of Jin and Wang concerning the conditions for a centre in certain cubic systems. Bull. London Math. Soc. 22 (1990) 5-12.

D. Cox, J. Little, D. O'Shea. Ideals, Varieties, and Algorithms: An Introduction to Computational Algebraic Geometry and Commutative Algebra. New York: Springer, 1997.

W. Decker, G.-M. Greuel, G. Pfister, H. Shönemann. Singular 3-1-6-A Computer Algebra System for Polynomial Computations. http://www.singular.uni-kl.de (2012).

W. Decker, S. Laplagne, G. Pfister, H.A. Schonemann. SINGULAR 3-1 library for computing the prime decomposition and radical of ideals, primdec.lib, 2010. 
H. Dulac. Détermination et intégration d'une certaine classe d'équations différentielles ayant pour point singulier un centre. Bull. Sci. Math. (2) 32 (1908) 230-252.

V. F. Edneral. Computer evaluation of cyclicity in planar cubic system, in Proceedings of the International Symposium on Symbolic and Algebraic Computation. New York, ACM Press, 1997, 305-309.

J.-P. Françoise, Y. Yomdin. Bernstein inequalities and applications to analytic geometry and differential equations. J. Functional Analysis 146 (1997) 185-205.

I. A. García, J. Llibre, S. Maza. Center cyclicity of a family of quartic polynomial differential system. Nonlinear Differ Equ. Appl. 23 (2016) 34pp, doi:10.1007/s00030-016-0388-8.

P. Gianni, B. Trager, G. Zacharias. Gröbner bases and primary decomposition of polynomials. J. Symbolic Comput. 6 (1988) 146-167.

J. Giné. On some open problems in planar differential systems and Hilberts 16th problem. Chaos, Solitons $\mathcal{E}$ Fractals 31 (2007) 1118-1134.

J. Giné, C. Christopher, M. Prešern, V.G. Romanovski, N.L. Shcheglova, The resonant center problem for a $2:-3$ resonant cubic Lotka-Volterra system, CASC 2012, Maribor, Slovenia, September 3-6, 2012. Lecture Notes in Computer Science 7442 (2012), 129-142.

A. S. Jarrah, R. Laubenbacher, and V. Romanovski. The Sibirsky component of the center variety of polynomial differential systems. Computer algebra and computer analysis (Berlin, 2001). J. Symbolic Comput. 35 (2003) 577-589.

X. Jin, D. Wang, On the conditions of Kukles for the existence of a centre. Bull. London Math. Soc. 22 (1990) 1-4.

J. Li. Hilbert's 16th problem and bifurcations of planar polynomial vector fields, Internat. J. Bifur. Chaos Appl. Sci Engrg. 13 (2003) 47-106.

J. Llibre, On the integrability of the differential systems in dimension two and of the polynomial differential systems in arbitrary dimension. J. Appl. Anal. Comput. 1 (2011) 33-52.

J. Llibre, X. Zhang, On the Darboux integrability of polynomial differential systems Qual. Theory Dyn. Syst. 11 (2012) $129-144$.

Y.-R. Liu, J.-B. Li, W. T. Huang. Singular point values, center problem and bifurcations of limit cycles of two-dimensional differential autonomous systems. Beijing: Science Press, 2008.

J. Llibre, C. Valls, Algebraic invariant curves and first integrals for Riccati polynomial differential systems, Proceedings of the American Mathematical Society 142 (2014) 3533-3543.

J. Llibre, C. Valls, Liouvillian first integrals for generalized Riccati polynomial differential systems, Advanced Nonlinear Studies 15 (2015) 951-961.

V. G. Romanovski, M. Prešern. An approach to solving systems of polynomials via modular arithmetics with applications. J. Comput. Appl. Math. 236 (2011) 196-208.

V. G. Romanovski, D. S. Shafer. The Center and cyclicity Problems: A computational Algebra Approach. Boston: Birkhauser, 2009.

K. S. Sibirskii. Algebraic Invariants of Differential Equations and Matrices. (Russian) Kishinev: Shtiintsa, 1976.

P.S. Wang, M.J.T. Guy, J.H. Davenport. P-adic reconstruction of rational numbers. SIGSAM Bull. 16 (1982), no. 2, 2-3. 